\title{
Uso da plataforma Mohid como ferramenta de apoio à tomada de decisão: um exemplo na região de Nova Friburgo.
}

\author{
R.S.G. Pontes ${ }^{1}$, G.S.L. Stefano ${ }^{1}$, W.R. Telles ${ }^{2}$, S.L.O. Gonzaga ${ }^{3}$, D.N. Brandão ${ }^{1}$, A.J. Silva Neto ${ }^{4}$ \\ ${ }^{1}$ Centro Federal de Educação Tecnológica Celso Suckow da Fonseca - CEFET-RJ \\ Maracanã, RJ - Brasil \\ ${ }^{2}$ Universidade Federal Fluminense - UFF \\ Santo Antônio de Pádua, RJ - Brasil \\ ${ }^{3}$ Universidade Federal de Lavras -UFLA \\ Lavras, MG - Brasil \\ ${ }^{4}$ Instituto Politécnico - UERJ \\ Nova Friburgo, RJ - Brasil \\ wtelles@id.uff.br, diego.brandaodcefet-rj.br
}

\begin{abstract}
Floods are defined as hazards caused by water flow abnormal behavior. In the present work, we evaluated a platform to study hydrological disasters caused by water flood in urban regions. MOHID (Modelagem Hidrodinâmica - Hydrological Modelling) computational platform is presented as a system to simulation the models of water flow and runoff in mountainous urban watersheds, allowing simulation of different scenarios. The importance of computational modeling and simulation as auxiliary tools for disaster risk reduction is discussed, showing MOHID as a system to decision support. Simulations results are presented for a real case-study with data from the Bengalas River watershed, in Nova Friburgo region.
\end{abstract}

Resumo. As inundações são definidas como desastres causados pelo comportamento anormal do fluxo de água. No presente trabalho, estamos particularmente interessados em enchentes que costumam acometer regiões urbanas. A plataforma computacional MOHID (Modelagem Hidrodinâmica) é apresentada como um sistema que permite a simulação dos modelos de escoamento de água em bacias hidrográficas, viabilizando a exploração de diferentes cenários sem prejuizos ambientais. Discutimos a importância da modelagem e simulação computacional como ferramentas auxiliares para redução do risco de desastres, demonstrando que tal sistema pode ser utilizado como uma ferramenta de apoio à tomada de decisão. Um estudo de caso é realizado com dados reais da bacia do rio Bengalas, na região de Nova Friburgo.

\section{Introdução}

Estudos envolvendo bacias hidrográficas são importantes, pois é neste local geográfico natural que verificamos a ocorrência de todos os componentes necessários ao crescimento e desenvolvimento da sociedade, além de permitirem a definição dos múltiplos usos dos recursos hídricos [ECOECO 1996]. A modelagem dessas regiões naturais envolve necessariamente o reconhecimento da distribuição espacial, temporal, das chuvas e 
das características de escoamento associadas. No que diz respeito ao escoamento em rios, estes podem ser matematicamente modelados em uma, duas ou três dimensões, utilizando para tanto descrições baseadas em Equações Diferenciais Parciais (EDPs) [Ogden et al. 2001, Alcoforado and Cirilo 2001, Fleenor and Jensen 2003].

A utilização de modelos computacionais para simular o comportamento de corpos hídricos permite que diversos cenários sejam avaliados sem que haja qualquer prejuízo ao meio ambiente. Diversos trabalhos utilizando essas abordagens vêm sendo desenvolvidos desde a década de 70 [Garbrecht and Martz 1999, Leal et al. 2014], além da criação de inúmeros softwares proprietários [Jacobs-Engineering-Group 2018, FLOW-3D 2018, FLO-2D-Software-Inc. 2018]. Nesse contexto, a plataforma MOHID fornece gratuitamente um arcabouço interessante de métodos e modelos para simulação de problemas envolvendo a área hidrológica [MARETEC 2012]. O MOHID (MOdelagem HIDrodinâmica) é um sistema de modelagem de problemas matemáticos envolvendo modelos hídricos desenvolvido pela MARATEC (Marine and Environmental Technology Research Center) do Instituto Superior Politécnico da Universidade de Lisboa e permite simulações em diferentes áreas da hidrodinâmica, desde que sejam conhecidas algumas informações reais da região de estudo (topografia, vazão, dentre outras) [MARETEC 2012].

Neste trabalho apresentamos o MOHID como um sistema de apoio a tomada de decisão, podendo auxiliar gestores públicos na identificação de riscos para a população, decorrentes de possíveis casos de inundações ou outros eventos hidrológicos. Como um sistema de simulação, ele permite que diferentes cenários sejam avaliados. Assim, possíveis situações de desastres podem ser preditas e medidas preventivas podem ser tomadas pelo poder público. Tais medidas vão desde obras de dragagem, passando pela contenção de encostas ou até mesmo a retirada emergencial da população para regiões seguras ou abrigos. Um estudo de caso também é apresentado. A partir das informações da topografia da cidade de Nova Friburgo, especificamente da Bacia do Rio Cônego confluente da Bacia do Rio Bengalas, e de informações pluviométricas, avaliamos um possível caso de enchente. A região de estudo tem grande importância para a cidade de Nova Friburgo, devido a ocorrência de inúmeros casos de enchentes, inclusive levando o município a declarar estado de calamidade pública no ano de 2011.

Este artigo está dividido em mais quatro seções. Na seção 2 apresentamos alguns trabalhos relacionados, nosso objetivo não é exaurir o assunto, mas demonstrar a importância que a utilização de simulação nos estudos sobre a enchentes vem tomando no mundo inteiro. A seção 3 descreve de forma objetiva a plataforma MOHID. Uma prova de conceito é apresentada na seção 4. Por fim, a seção 5 apresenta as considerações finais, bem como expectativas de trabalhos futuros.

\section{Trabalhos relacionados}

Nos últimos anos diversos estudos vem sendo realizados para investigar o risco de enchentes em diversos países do mundo. Na Europa destacam-se os trabalhos de [Ashley et al. 2005, Larsen et al. 2009, Willems 2013]. No Brasil destacam-se os trabalhos de [Speckhann et al. 2018] que avaliou os casos de enchentes na região sul do Brasil, especificamente a região do rio Itajaí. Já o trabalho de [Araújo et al. 2018] delimitou regiões de enchentes no vale do rio Uruguai, também no sul do Brasil. Por outro lado, os trabalhos de [Alfieri et al. 2016, Arnbjerg-Nielsen et al. 2015, Moore et al. 2016, 
Poussin et al. 2012] investigam as influências das mudanças climáticas devido ao fenômeno de aquecimento global nos casos de enchentes. Estudos mais gerais envolvem a relação dos índices pluviométricos com as enchentes [Olsson et al. 2009, Willems et al. 2012, Zahmatkesh et al. 2015], apresentando diferentes perspectivas sobre redes de drenagem.

Um simulador de enchentes em ambientes urbanos é apresentado por [Wang et al. 2018]. Nele os autores apresentam um simulador baseado em automatos celulares que integra as informações do sistema com dados oriundos de redes sociais, como vídeos e fotos, tudo em tempo real. Todavia, o simulador apresentado possui limites na dimensão da área a ser estudada, além de restrições referentes a uma compreensão analítica do modelo.

Diversos estudos com o MOHID vem sendo desenvolvidos para uma melhor compreensão da bacia do Rio Bengalas na região serrana do Rio de Janeiro [Telles et al. 2012, W.R.Telles et al. 2013, Telles 2014], inclusive para os eventos catástroficos de janeiro de 2011[Telles et al. 2017a, Telles et al. 2017b]. Todavia, tais estudos referem-se principalmente aos modelos matemáticos e aos métodos de simulação, estudos demonstrando a potencialidade do MOHID como um sistema de apoio a tomada de decisão ainda são escassos. Além de ser um ferramenta robusta para solução de problemas hidrodinâmicos, pois permite simulações em regiões bem abrangentes com modelos uni,bi e tridimensionais, o MOHID utiliza abordagens baseadas em modelos descritos por equações diferenciais, assim estudos analíticos tornam-se possíveis.

\section{Plataforma MOHID}

Em 1985 a MARETEC (Marine and Environmental Technology Research Center) do Instituto Superior Politécnico da Universidade de Lisboa iniciou o desenvolvimento de um software de modelagem matemática para problemas envolvendo fenômenos hidrológicos [MARETEC 2012]. O sistema era desenvolvido em linguagem FORTRAN, inicialmente para o sistema operacional Windows.

Nos anos seguintes o sistema ganhou uma interface gráfica e hoje é constituído basicamente por duas: MOHID GUI e MOHID GIS. A primeira interface concentra módulos responsáveis pela simulação e análise de estuários, bacias hidrográficas, etc. E a segunda é responsável pela parte geográfica, isto é, ela consiste em um sistema de informações geográficas que contém os dados referentes a digitalização dos dados reais de terreno, topografia e etc. Somente a segunda consegue dar um significado aos dados produzidos pelos modelos numéricos [W.R.Telles et al. 2013].

\section{Prova de Conceito}

Nova Friburgo é uma cidade localizada na região serrana do Estado do Rio de Janeiro que ocupa uma área total de aproximadamente $933 \mathrm{~km}^{2}$ (Figura 1). Dentro dessa cidade a bacia do rio Bengalas ocupa uma área de $192 \mathrm{~km}^{2}$, sendo localizada na zona mais urbanizada da cidade. O rio Bengalas é formado pela confluência de outros dois rios, a saber, rio Santo Antonio e rio Cônego. O estudo desses rios é fundamental para o funcionamento da cidade que já foi inúmeras vezes castigada por diversas enchentes. A última ocorrida em 2011 devastou toda a cidade, levando a falência de inúmeras industrias têxteis, além da diminuição do fluxo turístico na região. 


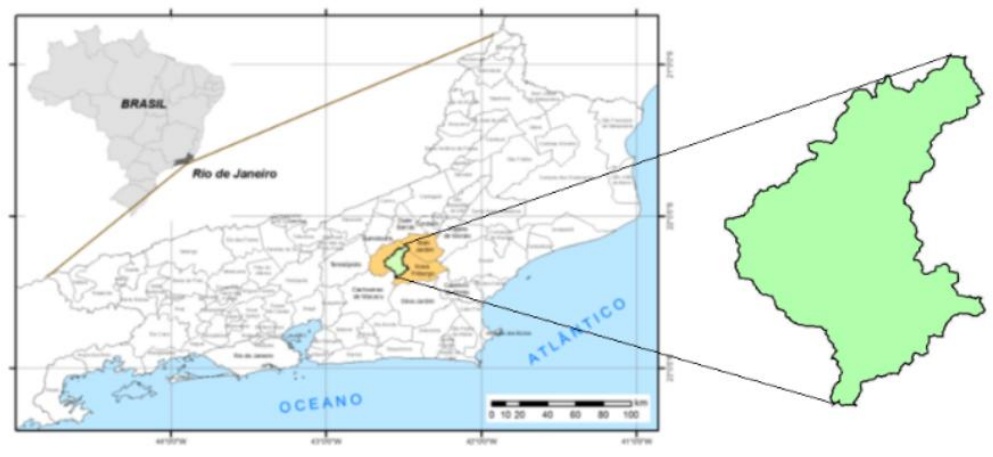

Figura 1. Região de Nova Friburgo.

\subsection{Geração dos mapas}

O processo de digitalização dos dados referentes ao rio Bengalas envolve a conversação e rasterização dos dados obtidos. No caso do MOHID a delimitação da bacia hidrográfica pode ser realizada tanto por um ponto de saída da rede de drenagem quanto pela área mínima de onde se deseja formar os canais. A Figura 2 apresenta a rede de drenagem obtida por esse processo do mapa digital do rio Cônego.

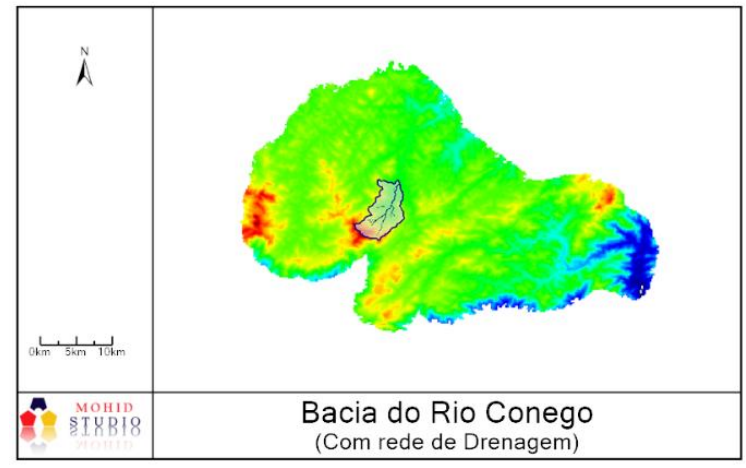

Figura 2. Rede de drenagem do rio Cônego.

A Figura 3 apresenta as sub-bacias do rio Bengalas obtidas pelo MOHID. Os polígonos obtidos nesse processo demonstram uma boa semelhança em relação aos limites da bacia do rio Bengalas.

O estudo de caso apresentado utiliza dados de precipitação do mês de novembro de 2011 da Bacia do Cônego (Figura 2), em especial da madrugada de 13 para 14 daquele mês. A escolha da data posterior ao desastre ocorrido em janeiro de 2011 deve-se aos parâmetros mensurados para esta Bacia em estudo apresentado por [Telles et al. 2012]. As informações de profundidade do canal mensuradas referem-se ao ponto demarcado na Figura 4.

\subsection{Resultados da Simulação}

A Figura 5 apresenta o resultado da simulação realizada no MOHID. Nela são comparados os índices pluviométricos com a coluna da água após um período de chuva intensa. Esse resultado demonstra que diversos cenários podem ser simulados e com isso pode 


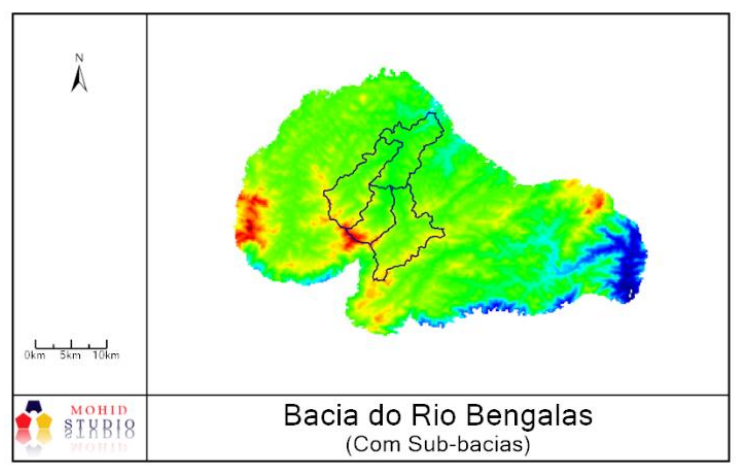

Figura 3. Sub-bacia do rio Bengalas.

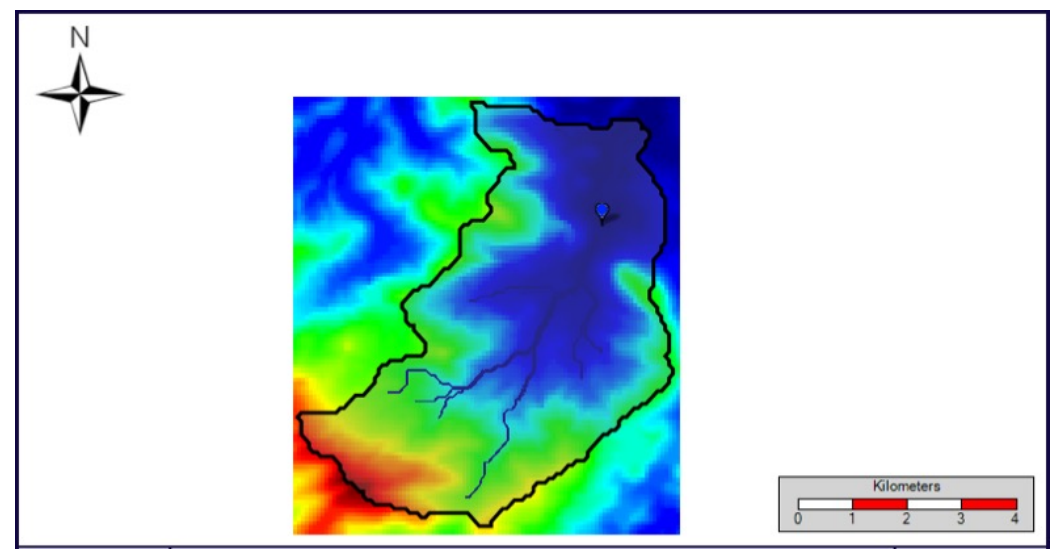

Figura 4. Ponto onde os dados pluviométricos foram coletados.

ser identificado se o nível a ser alcançado pela água poderá representar riscos para a população que vive a beira do corpo hídrico.

O MOHID permite que o comportamento evolutivo do corpo hídrico seja avaliado também. A Figura 6 mostra a evoluação no nível da água em diversos instantes de tempo posteriores ao período chuvoso. Observe que o MOHID tem potencial para permitir que a partir da simulação de diferentes cenários seja criado um sistema de alerta para enchentes. Sempre que os índices pluviométricos alcançarem um determinado patamar a população poderia ser avisada a priori para procurar uma região onde se mantivesse em segurança.

\section{Considerações Finais}

O MOHID propõem como uma alternativa consolidada para solução de EDPs utilizadas na análise dos índices pluviométricos em rios com base nas precipitações que assolam ou podem assolar a região. Com isso ele permite, além de entender o comportamento do elemento hídrico após o encadeamento de chuvas, também, estimá-lo mediante a variação das características do mesmo e assim prever o impacto de uma obra ou de uma mudança nos índices de precipitação antes que eles ocorram, podendo assim prevenir possíveis catástrofes decorrentes de obras ou crescimento previsto das chuvas.

Como trabalho futuro vislumbra-se a possibilidade de estudo sobre o rio Sarapuí, que corta cinco municípios da Baixada Fluminense, no Rio de Janeiro. Para isso dados topográficos precisam ser obtidos junto ao IBGE ou as autoridades municipais/estaduais, 


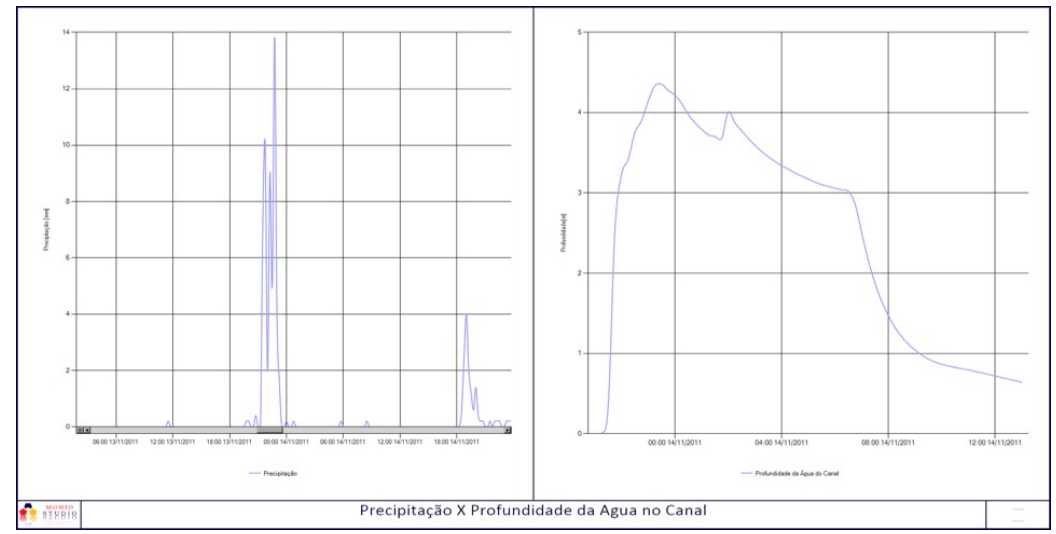

Figura 5. Simulação comparando o indice pluviométrico com o aumento da coluna de água.

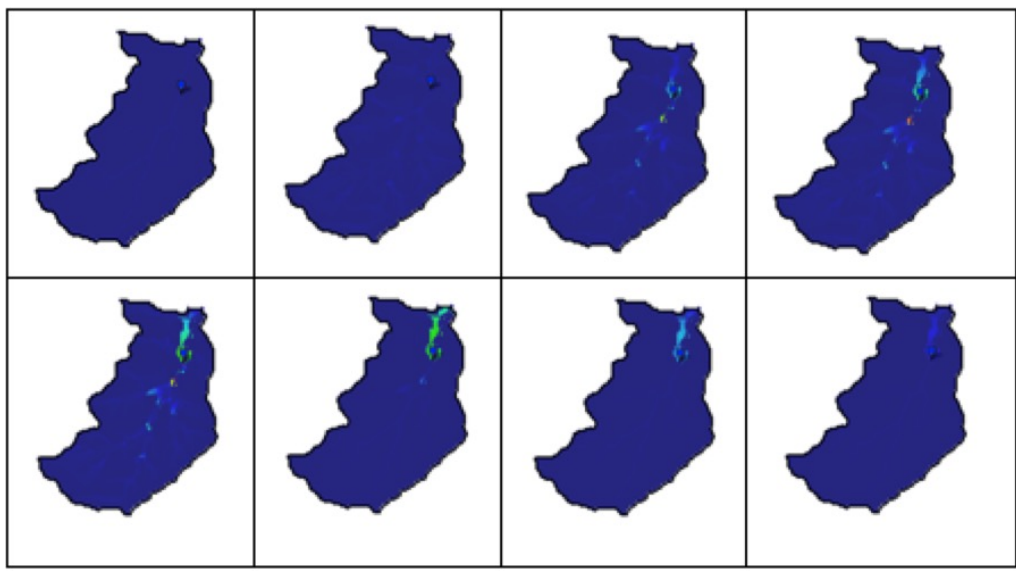

Figura 6. Evolução do escoamento da água na bacia hidrográfica. As figuras na parte superior representam as primeiras horas do aumento de fluxo de água. A primeira figura na parte inferior esquerda representa o ápice do processo que se encerra na última figura a direita da parte inferior.

os parâmetros hidráulicos precisam ser adquiridos ou, caso não conhecidos, determinados numericamente.

\section{Agradecimentos}

Os autores agradecem o suporte financeiro da FAPERJ - Fundação de Amparo à Pesquisa do Estado do Rio de Janeiro, do CNPq - Conselho Nacional de Desenvolvimento Científico e Tecnológico e da CAPES - Coordenação de Aperfeiçoamento de Pessoal de Nível Superior.

\section{Referências}

Alcoforado, R. and Cirilo, J. (2001). Sistema de suporte à decisão para análise, previsão e controle de inundações. Revista Brasileira de Recursos Hídricos.

Alfieri, L., Feyen, L., and Di Baldassarre, G. (2016). Increasing flood risk under climate change: a pan-european assessment of the benefits of four adaptation strategies. Climatic Change, 136:507-521. 
Araújo, P. V. N., Amaro, V. E., Silva, R. M., and Lopes, A. B. (2018). Delimitation of flood areas based on calibrated dem and geoprocessing: Case study on uruguay river, itaqui city, southern brazil. Natural Hazards and Earth System Sciences Discussions, 2018:1-22.

Arnbjerg-Nielsen, K., Leonardsen, L., and Madsen, H. (2015). Evaluating adaptation options for urban flooding based on new highend emission scenario regional climate model simulations. Clim. Res., 64:73-84.

Ashley, R. M., Balmforth, D. J., Saul, A. J., and Blanskby, J. D. (2005). Flooding in the future - predicting climate change, risks and responses in urban areas. Water Sci. Technol., 52:265-273.

ECOECO (1996). Bacias hidrográficas: Nova gestão de recursos hídricos. I Encontro Nacional da ECOECO.

Fleenor, W. and Jensen, M. (2003). Evaluation of numerical models hec-ras and dhi-mike 11. Journal of Hydrologic Engineering.

FLO-2D-Software-Inc. (1986-2018). Flo-2d software. https://www.flo-2d. $\mathrm{com} /$.

FLOW-3D (1980-2018). Flash flood simulation. https://www. flow3d.com/ flash-flood-simulation/.

Garbrecht, J. and Martz, L. (1999). Digital elevation model issues in water resources modeling. ESRI, Users Conference.

Jacobs-Engineering-Group (2015-2018). Flood modeller. https: / / www . floodmodeller.com.

Larsen, A. N., Gregersen, I. B., Christensen, O. B., Linde, J. J., and Mikkelsen, P. S. (2009). Potential future increase in extreme one-hour precipitation events over europe due to climate change. Water Sci. Technol., 60:2205-2216.

Leal, T., Moura, C., Isaac, C., and Burgarelli, D. (2014). Aplicação de técnicas de análise numérica a um modelo de escoamento de água em áreas amazônicas alagáveis. Anais do XXXV Congresso Nacional de Matemática Aplicada e Computacional.

MARETEC (2012). Mohid: Descrição do mohid. Boletim do Observatório Ambiental Alberto Ribeiro Lamego, 1.

Moore, T. L., Gulliver, J. S., Stack, L., and Simpson, M. H. (2016). Stormwater management and climate change: vulnerability and capacity for adaptation in urban and suburban contexts. Climatic Change, 138:491-504.

Ogden, F., Garbrecht, J., DeBarry, P., and Johnson, L. (2001). Gis and distributed watershed models. ii: modules, interfaces, and models. Journal of Hydrologic Engineering.

Olsson, J., Berggren, K., Olofsson, M., and Viklander, M. (2009). Applying climate model precipitation scenarios for urban hydrological assessment: A case study in kalmar city, sweden. Atmos. Res., 92:364-375. 
Poussin, J. K., Bubeck, P., Aerts, J. C. J. H., and Ward, P. J. (2012). Potential of semistructural and non-structural adaptation strategies to reduce future flood risk: case study for the meuse. Nat. Hazards Earth Syst. Sci., 12:3455-3471.

Speckhann, G. A., Chaffe, P. L. B., Goerl, R. F., de Abreu, J. J., and Flores, J. A. A. (2018). Flood hazard mapping in southern brazil: a combination of flow frequency analysis and the hand model. Hydrological Sciences Journal, 63(1):87-100.

Telles, W. (2014). Previsão do comportamento hidráulico de um rio com base na estimativa de coeficientes que controlam seu escoamento. estudo de caso: Rio bengalas, nova friburgo-rj. Tese de Doutorado. Universidade do Estado do Rio de Janeiro.

Telles, W., Silva Neto, A., and Rodrigues, P. (2012). Avaliação do sistema de modelagem mohid na delimitação de bacias hidrográficas. Congresso de Matemática Aplicada e Computacional., pages 589-592.

Telles, W., Silva Neto, A., and Rodrigues, P. (2017a). Calibração automática da plataforma mohid empregando um método estocástico de otimização e dados reais de um evento climático extremo em nova friburgo-rj: parte 1 - preparação do modelo digital do terreno e aquisição dos dados experimentais de precipitação e nível. Revista Internacional de Métodos Numéricos para Cálculo y Diseño en Ingeniería., pages 164-170.

Telles, W., Silva Neto, A., and Rodrigues, P. (2017b). Calibração automática da plataforma mohid empregando um método estocástico de otimização e dados reais de um evento climático extremo em nova friburgo-rj: parte 2 - análise de sensibilidadee estimativa de parâmetros hidrológicos. Revista Internacional de Métodos Numéricos para Cálculo y Diseño en Ingeniería., pages 204-211.

Wang, Y., Chen, A., Fu, G., Djordjević, S., Zhang, C., and Savić, D. A. (2018). An integrated framework for high-resolution urban flood modelling considering multiple information sources and urban features. Environmental Modelling Software, 107:8595.

Willems, P. (2013). Revision of urban drainage design rules after assessment of climate change impacts on precipitation extremes at uccle. belgium. J. Hydrol., 496:166-177.

Willems, P., Arnbjerg-Nielsen, K., Olsson, J., and Nguyen, V. T. V. (2012). Climate change impact assessment on urban rainfall extremes and urban drainage: Methods and shortcomings. Atmos. Res., 103:106-118.

W.R.Telles, Rodrigues, P., and da Silva Neto, A. J. (2013). Utilização de sistemas de informações geográficas na delimitação de bacias hidrográficas. Boletim do Observatório Ambiental Alberto Ribeiro Lamego, 1.

Zahmatkesh, Z., Karamouz, M., Goharian, E., and Burian, S. J. (2015). Analysis of the effects of climate change on urban storm water runoff using statistically downscaled precipitation data and a change factor approach. J. Hydrol. Eng., 20:1. 